\title{
Taxonomic Study of the Genus Brachybacterium: Proposal of Brachybacterium conglomeratum sp. nov., nom. rev., Brachybacterium paraconglomeratum sp. nov., and Brachybacterium rhamnosum sp. nov.

\author{
MARIKO TAKEUCHI, ${ }^{*}$ CHENG-XIANG FANG,$\dagger$ AND AKIRA YOKOTA \\ Institute for Fermentation, Osaka, Yodogawa-ku, Osaka 532, Japan
}

\begin{abstract}
On the basis of morphological, physiological, and chemotaxonomic characteristics and DNA-DNA hybridization data, we describe three new Brachybacterium species: Brachybacterium conglomeratum sp. nov., nom. rev. (type strain, IFO 15472); Brachybacterium paraconglomeratum sp. nov. (type strain, IFO 15224); and Brachybacterium rhamnosum sp. nov. (type strain, IFO 15203). These new species include some previously misclassified strains and an isolate obtained from corn steep liquor. Although $B$. conglomeratum and $B$. paraconglomeratum are similar in their physiological characteristics, they can be differentiated on the basis of their cellular fatty acid patterns and low levels of DNA-DNA hybridization. B. rhamnosum is characterized by the presence of large amounts of rhamnose in its cell wall.
\end{abstract}

The genus Brachybacterium was established by Collins et al. (2). This genus is characterized by the following chemotaxonomic features: the peptidoglycan type is the meso-diaminopimelic acid (DAP)-D-Glu ${ }_{2}$ type (variation A4y) (15); the principal menaquinone is MK-7; mycolic acids are absent; and the $\mathrm{G}+\mathrm{C}$ content of the DNA is 68 to $72 \mathrm{~mol} \%$. Until now, the genus Brachybacterium has been represented by two species, Brachybacterium faecium (2) and Brachybacterium nesterenkovii (5), but it has been suggested that several other bacterial strains belong to this genus (16). Bogdanovsky et al. (1) determined the peptidoglycan structures of "Micrococcus conglomeratus" strains and Micrococcus roseus (formerly Staphylococcus lactis) and found that the peptidoglycan type of these bacteria is the meso-DAP-D-Glu ${ }_{2}$ type (variation A4 $\gamma$ ), a type which has been found only in the genus Brachybacterium. Nogina et al. (11) suggested that these organisms should be assigned to the genus Brachybacterium, although their taxonomic positions at the species level were not certain. In addition, one strain isolated in our laboratory had the morphological, biochemical, and chemotaxonomic characteristics peculiar to the genus Brachybacterium.

In this paper, we describe the phenotypic and chemotaxonomic characteristics of the strains mentioned above, and on the basis of these characteristics and the results of a DNADNA hybridization study, we describe three new Brachybacterium species.

\section{MATERIALS AND METHODS}

Microorganisms and cultures. The strains which we used are listed in Table 1. B. faecium NCIB $9860^{\mathrm{T}}$ ( $\mathrm{T}=$ type strain) NCIB 9859, and NCIB 9861 were obtained from the National Collection of Industrial Bacteria, eight strains of " $M$. conglomeratus" and one Brachybacterium sp. strain were obtained from the Czech Collection of Microorganisms, and $M$. roseus NCIC 15264 was obtained from the National Collection of Type Cultures, Central Public Health Laboratory, London, England. Brachybacterium sp. strain H-6S ${ }^{\mathrm{T}}$ (= IFO $15203^{\mathrm{T}}$ ) was isolated in our laboratory from commercially available corn steep liquor. All strains were cultured at $28^{\circ} \mathrm{C}$ with aerobic shaking in a peptone-yeast extract

* Corresponding author. Mailing address: Institute for Fermentation, Osaka, 17-85, Juso-honmachi 2-chome, Yodogawa-ku, Osaka 532, Japan. Phone: 81-6-300-6555. Fax: 81-6-300-6814.

† Present address: College of Life Sciences, Wuhan University, Wuhan 430072, People's Republic of China. medium supplemented with brain heart infusion (PY-BHI medium), which contained $1 \%$ peptone, $0.2 \%$ yeast extract, $0.5 \%$ brain heart infusion (Difco Laboratories), $0.2 \% \mathrm{NaCl}$, and $0.2 \%$ glucose ( $\mathrm{pH} 7.0$ ). Cells were harvested by centrifugation, washed with water, and then lyophilized.

Morphological characteristics. Cell morphology was determined by using cells grown on yeast extract-peptone-brain heart infusion agar (1\% yeast extract, $0.5 \%$ peptone, $0.2 \%$ brain heart infusion, $2 \%$ agar [pH 7.0]). Each sample used for scanning electron microscopy with a model JSM 5400 scanning electron microscope (JEOL, Ltd., Tokyo, Japan) was prepared by fixing cells with $1 \%$ glutaraldehyde and then dehydrating them with a graded acetone series and then in a Hitachi model HCP-2 critical point drying apparatus.

Determination of phenotypic characteristics. Cultures were grown on PY-BHI medium containing $1.5 \%$ agar. Motility was determined with a light microscope by the hanging drop method. All tests were performed at $28^{\circ} \mathrm{C}$. Catalase activity was determined by monitoring bubble formation in a $3 \%$ hydrogen peroxide solution. Oxidase activity was determined by monitoring the oxidation of $1 \%$ tetramethyl- $p$-phenylenediamine on filter paper. Acid production from carbohydrates was determined in medium containing $1.0 \%$ peptone, $0.5 \% \mathrm{NaCl}, 0.003 \%$ bromcresol purple, and $0.5 \%$ carbohydrate $(\mathrm{pH} \mathrm{7.2).} \mathrm{Assimilation} \mathrm{of} \mathrm{carbohy-}$ drates and organic acids was determined in medium containing $0.5 \%$ carbohydrate or $0.5 \%$ organic acid (sodium salt), $0.1 \% \mathrm{NH}_{4} \mathrm{NO}_{3}, 0.1 \% \mathrm{KH}_{2} \mathrm{PO}_{4}, 0.05 \%$ $\mathrm{MgSO}_{4} \cdot 7 \mathrm{H}_{2} \mathrm{O}$, and $0.02 \% \mathrm{KCl}(\mathrm{pH} 7.2)$.

Other phenotypic characteristics, production of indole and $\mathrm{H}_{2} \mathrm{~S}$, the VogesProskauer test reaction, nitrate reduction, and hydrolysis of starch, gelatin, casein, and esculin were determined by the methods described by Cowan and Steel (3).

Analyses of chemotaxonomic characteristics. Cell walls were prepared from ca. $500 \mathrm{mg}$ (dry weight) of cells by mechanical disruption with an ultrasonic oscillator and were purified as described by Schleifer and Kandler (15). The amino acid compositions of complete wall hydrolysates were determined by high-performance liquid chromatography (HPLC) (19), as described by Yokota et al. (20). For the cell wall sugar analysis, cell walls were hydrolyzed with $2 \mathrm{~N}$ $\mathrm{HCl}$ at $100^{\circ} \mathrm{C}$ for $2 \mathrm{~h}$, dried in vacuo, and then analyzed by HPLC by the method of Mikami and Ishida (8). Glycolate tests were performed by the method of Uchida and Aida (18). Fatty acids were extracted from dry cells $(50 \mathrm{mg})$ by acid methanolysis, and fatty acid contents were determined by gas-liquid chromatography as described previously (17). Free lipids were extracted from dry cells (100 $\mathrm{mg}$ ) and purified by the method of Minnikin et al. (10), and free lipid contents were determined by two-dimensional thin-layer chromatography, using Kieselgel $60 \mathrm{~F}_{254}$ plates (Merck, Darmstadt, Germany). Lipid spots were revealed by spraying the chromatograms with $10 \%$ molybdophosphoric acid in ethanol and then heating them at $150^{\circ} \mathrm{C}$ for $15 \mathrm{~min}$. Specific spray reagents for lipid phosphate, $\alpha$-glycol (periodate-Schiff reagent), sugars ( $\alpha$-naphthol), and free amino groups (ninhydrin in water-saturated butanol) were also used. Mycolic acids were analyzed by the method of Minnikin et al. (9). Menaquinones were extracted from $200 \mathrm{mg}$ of dry cells with chloroform-methanol (2:1, vol/vol), purified by thin-layer chromatography, extracted with diethyl ether, dried under a nitrogen stream, and then analyzed by HPLC.

DNA-DNA hybridization. DNA was obtained by the method of Saito and Miura (13). The G+C content of DNA was determined by HPLC by the method of Mesbah et al. (7) after treatment with $\mathrm{P}_{1}$ nuclease and alkaline phosphatase. DNA-DNA hybridization was carried out fluorometrically in microdilution wells by using biotinylated DNA (4). 


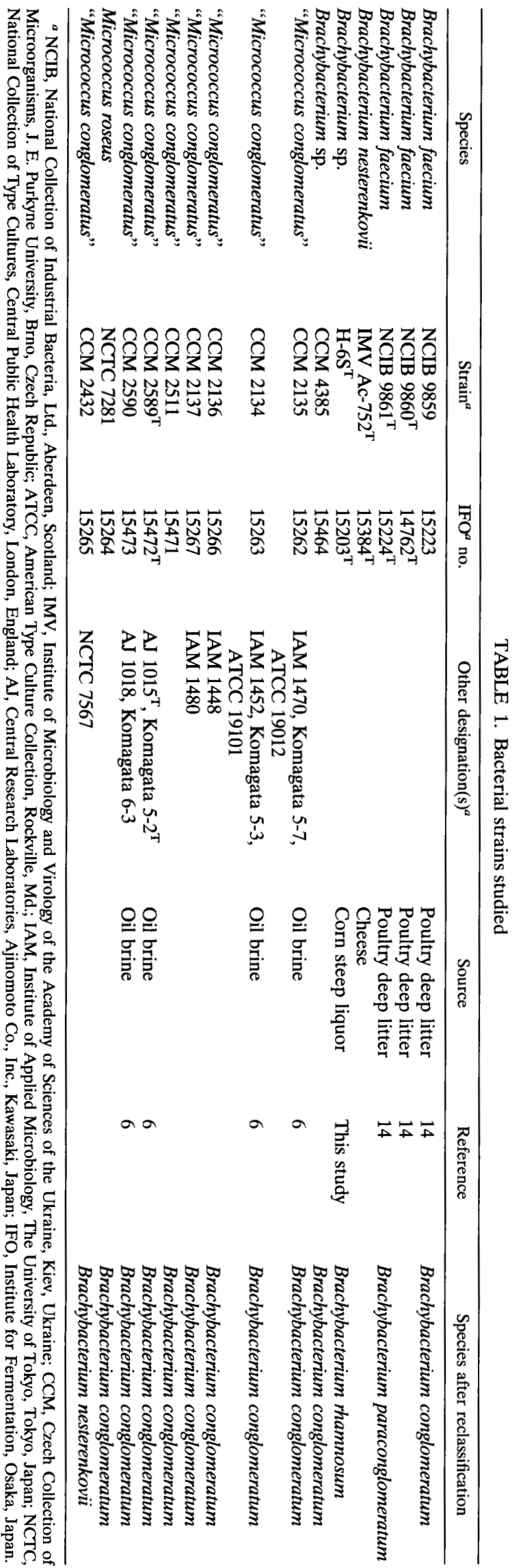

\section{RESULTS}

Morphological, physiological, and biochemical characteristics. All of the organisms which we examined had similar morphological properties. In the stationary growth phase, cultures consisted mainly of coccoid cells that were 0.5 to $1 \mu \mathrm{m}$ in diameter, but 6 to $8 \mathrm{~h}$ after the organisms were transferred to fresh medium, the cells were oval or short rods that were up to $2 \mu \mathrm{m}$ long (Fig. 1). Some cells exhibited a V-shaped arrangement. The cells were gram positive, not acid fast, nonmotile, and nonsporing. Colonies on PY-BHI agar were convex and had regular edges, and their surfaces were smooth or rough. Colonies were yellow, yellow-green, pale brown, or greyish white on PY-BHI agar. As shown in Table 2, all strains gave positive results in the tests for catalase activity, growth in broth containing 0 to $7.5 \% \mathrm{NaCl}$, hydrolysis of esculin and starch, nitrate reduction, and formation of acid from L-arabinose and D-glucose, while all strains gave negative results in tests for oxidase activity, hydrolysis of Tween 20 and Tween 40, and formation of acid from mannitol and xylitol.

Chemical characteristics. The chemical characteristics of the strains which we studied are summarized in Tables 3 and 4. All strains contained menaquinone MK-7, and the DNA base compositions of the strains ranged from 70.2 to $72.6 \mathrm{~mol} \%$. All strains contained high levels of glycolate in the glycan moieties of their cell walls, which suggests that muramic acid occurred in the $N$-glycolyl form rather than the more common $N$-acetyl form. Mycolic acids were absent. Galactose and glucose were common cell wall sugars in all strains, while the cell wall preparations of $B$. nesterenkovii IMV Ac- $752^{\mathrm{T}}$ and " $M$. conglomeratus" CCM 2432 also contained small amounts of rhamnose. Brachybacterium sp. strain $\mathrm{H}-6 \mathrm{~S}^{\mathrm{T}}$ contained rhamnose as a major component of its cell wall. 6-Deoxytalose was detected in the cell wall preparation of the type strain of $B$. faecium, strain NCIB 9860. The amino acids in the cell wall hydrolysates of all of the strains except $M$. nesterenkovii IMV Ac-752 $2^{\mathrm{T}}$ and " $M$. conglomeratus" CCM 2432 were meso-DAP, alanine, glutamic acid, and glycine (molar ratio, 1:2:2 3:1), whereas the amino acids in the cell wall hydrolysates of strains IMV Ac- $752^{\mathbf{T}}$ and CCM 2432 were meso-DAP, alanine, and glutamic acid (molar ratio, 1:2:2 3); glycine was not present in the cell wall hydrolysates of strains IMV Ac- $752^{\mathrm{T}}$ and CCM 2432. On the basis of the molar ratios of the amino acids we suggest that the peptidoglycan structure type of all strains is type A4 $\gamma$. Figure 2 shows the results of our two-dimensional thin-layer chromatography analysis of the polar lipids of representative strains. The diagnostic phospholipid patterns indicated that diphosphatidylglycerol, phosphatidylglycerol, glycolipids, and unidentified phosphoglycolipids (ninhydrin-positive unknown phosphoglycolipid or ninhydrin-negative unknown phosphoglycolipid) were present in the strains which we studied. In this analysis we identified six different phospholipid profiles (profiles A through F). As shown in Fig. 2, profile B, which was found in $B$. nesterenkovii IMV Ac-752 $2^{\mathrm{T}}$, was composed of three lipids, diphosphatidylglycerol, glycolipid, and phosphatidylglycerol. Profile A, which was found in B. faecium NCIB $9860^{\mathrm{T}}$, contained a ninhydrin-negative unknown phosphoglycolipid in addition to the lipids found in profile $B$. Profile C, which was found in B. faecium NCIB 9861, contained a ninhydrin-positive unknown phosphoglycolipid in addition to the lipids found in profile A. Like profile A, profile D was composed of four lipids, diphosphatidylglycerol, a ninhydrinnegative unknown phosphoglycolipid, glycolipid, and phosphatidylglycerol, but two kinds of glycolipids were present in profile $\mathrm{D}$ and the positions of these components on thin-layer chromatography plates were different. In Brachybacterium con- 

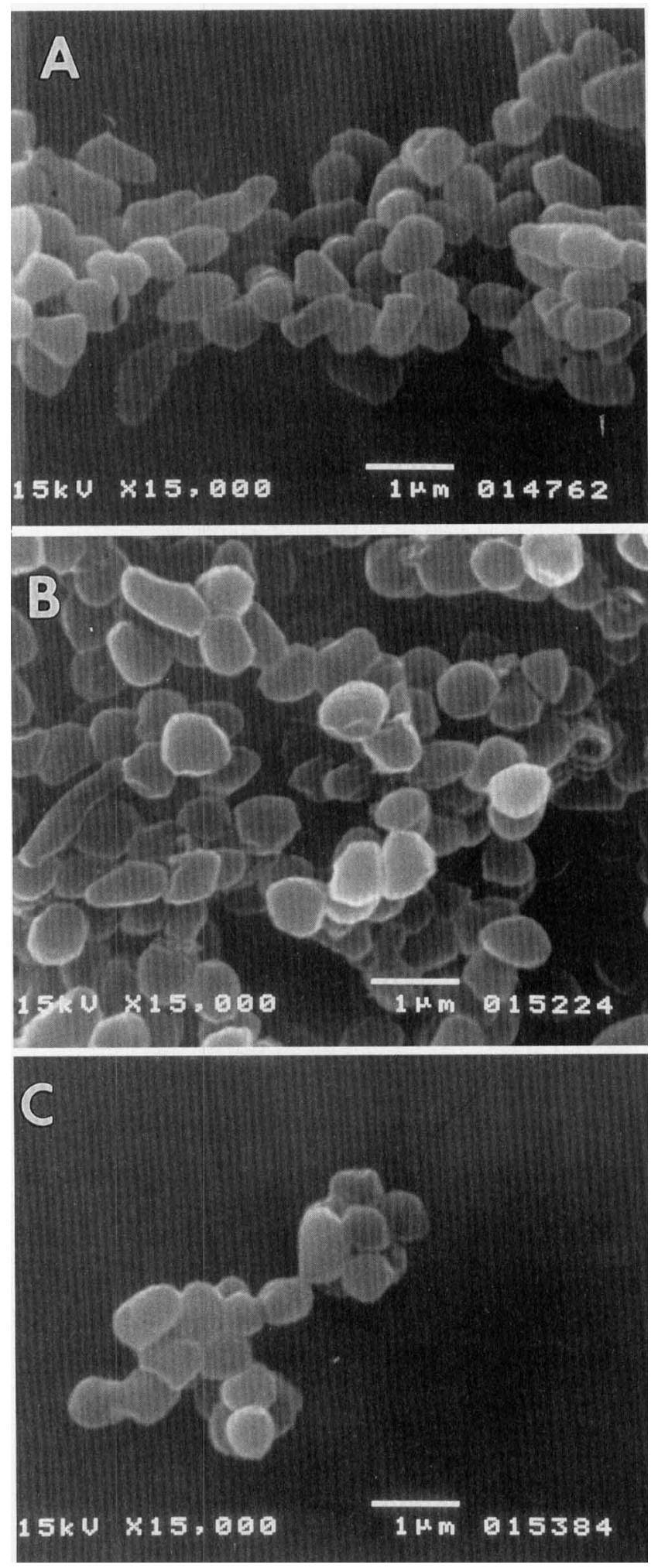

glomeratum, two profiles (profiles E and F) were found. Both profile $\mathrm{E}$ and profile $\mathrm{F}$ were composed of five lipids, but two kinds of ninhydrin-positive unknown phosphoglycolipids and two types of glycolipids were found in profiles $E$ and $F$, respectively. Therefore, the $B$. conglomeratum (profiles $\mathrm{E}$ and F) and Brachybacterium paraconglomeratum (profile C) could
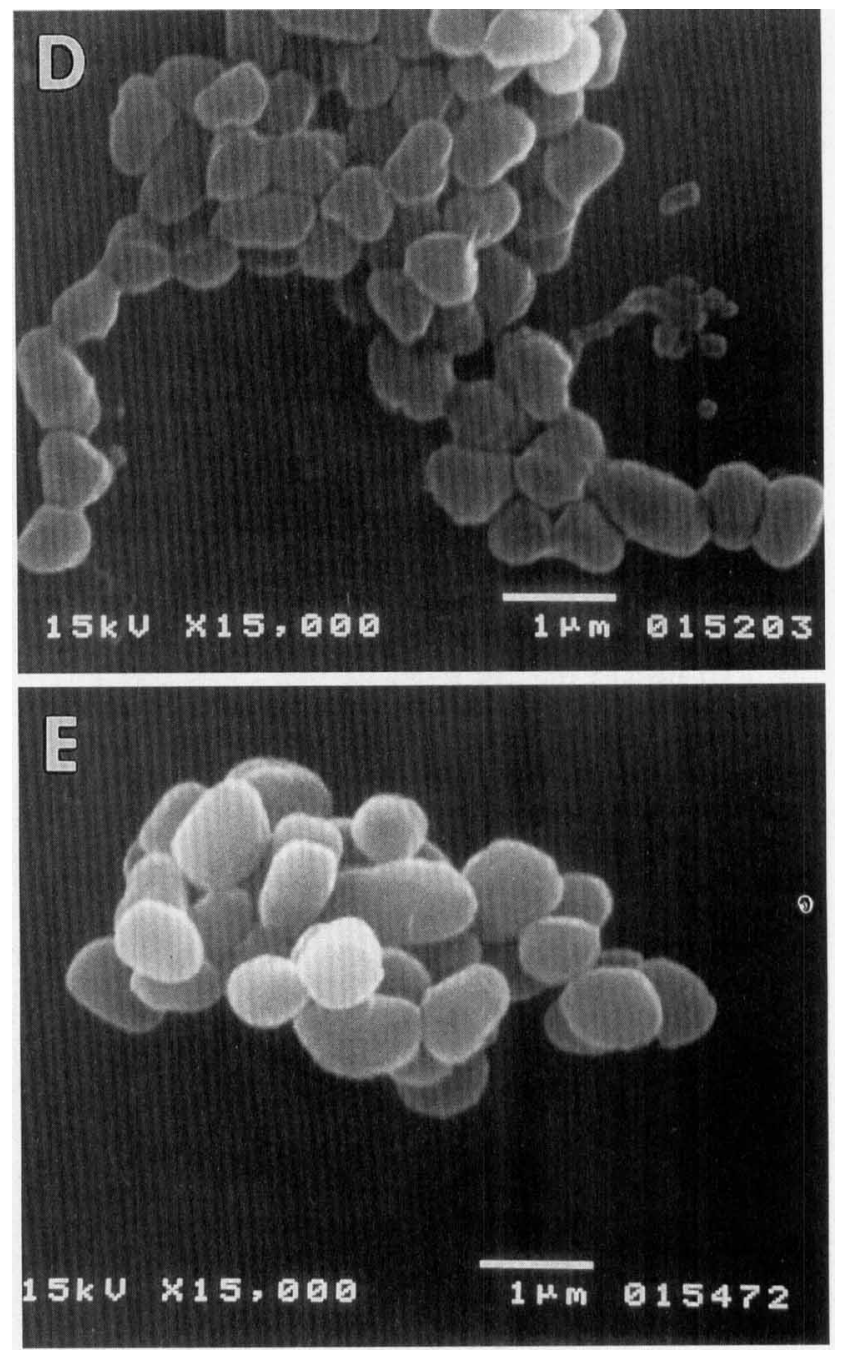

FIG. 1. Scanning electron micrographs of Brachybacterium strains grown on PY-BHI agar for 1 day. (A) B. faecium NCIB $9860^{\mathrm{T}}$. (B) B. paraconglomeratum NCIB $9861^{\mathrm{T}}$. (C) B. nesterenkovii IMA Ac-752 $2^{\mathrm{T}}$. (D) B. rhamnosum $\mathrm{H}-6 \mathrm{~S}^{\mathrm{T}}$. (E) B. conglomeratum $\mathrm{CCM} 2589^{\mathrm{T}}$.

not be clearly differentiated on the basis of their phospholipid patterns. All of the strains produced fatty acid profiles which consisted primarily of anteiso methyl-branched acids, including 12-methyltetradecanoic (anteiso- $\mathrm{C}_{15: 0}$ ) and 14-methylhexadecanoic (anteiso- $\mathrm{C}_{17: 0}$ ) (Table 4). The results of a cluster analysis based on fatty acid compositions are shown in Fig. 3; this analysis revealed that $B$. nesterenkovii and Brachybacterium rhamnosum strains belong to a complex together with $B$. conglomeratum strains, but that $B$. faecium and $B$. paraconglomeratum strains belong on different branches.

On the basis of morphological, physiological, and chemotaxonomic characteristics, all of the strains were identified as members of the genus Brachybacterium.

DNA-DNA hybridization. On the basis of DNA-DNA hybridization values (Table 5) we separated the strains which we studied into five distinct DNA relatedness groups. The $B$. faecium strains (NCIB 9860 ${ }^{\mathrm{T}}$, NCIB 9859, and NCIB 9861) exhibited low levels of DNA-DNA homology with each other (13 to $49 \%$ ). The type strain of B. faecium, NCIB 9860, and strain NCIB 9861 did not exhibit a high level of homology with any other strain tested, but $B$. faecium NCIB 9859 exhibited high levels of homology with nine strains of " $M$. conglomeratus" (CCM 2135, CCM 2134, CCM 2136, CCM 2137, CCM 
TABLE 2. Phenotypic characteristics of Brachybacterium strains

\begin{tabular}{|c|c|c|c|c|c|c|c|c|c|c|c|c|c|c|c|}
\hline \multirow{2}{*}{ Characteristic } & \multirow{2}{*}{$\begin{array}{l}\text { B. faecium } \\
\text { NCIB } \\
9860^{\mathrm{T}}\end{array}$} & \multicolumn{2}{|c|}{$\begin{array}{l}\text { B. nesterenkovii } \\
\text { strains }\end{array}$} & \multirow{2}{*}{$\begin{array}{l}\text { B. paracon- } \\
\text { glomeratum } \\
\text { NCIB } 9861^{\mathrm{T}}\end{array}$} & \multirow{2}{*}{$\begin{array}{l}\text { B. rham- } \\
\text { nosum } \\
\mathrm{H}-6 \mathrm{~S}^{\mathrm{T}}\end{array}$} & \multicolumn{10}{|c|}{ B. conglomeratum strains } \\
\hline & & $\begin{array}{c}\text { IMV } \\
{\text { Ac- } 752^{\mathrm{T}}}^{2}\end{array}$ & $\begin{array}{l}\text { CCM } \\
2432\end{array}$ & & & $\begin{array}{l}\text { NCIB } \\
9859\end{array}$ & $\begin{array}{l}\mathrm{CCM} \\
4385\end{array}$ & $\begin{array}{l}\mathrm{CCM} \\
2135\end{array}$ & $\begin{array}{l}\text { CCM } \\
2134\end{array}$ & $\begin{array}{l}\mathrm{CCM} \\
2136\end{array}$ & $\begin{array}{l}\mathrm{CCM} \\
2137\end{array}$ & $\begin{array}{l}\mathrm{CCM} \\
2511\end{array}$ & $\begin{array}{l}\mathrm{CCM} \\
2589^{\mathrm{T}}\end{array}$ & $\begin{array}{l}\text { CCM } \\
2590\end{array}$ & $\begin{array}{l}\text { NCTC } \\
7281\end{array}$ \\
\hline Growth rate $^{a}$ & $\mathrm{~s}$ & $\mathbf{R}$ & $\mathbf{R}$ & $\mathrm{S}$ & $\mathbf{R}$ & $\mathbf{R}$ & $\mathbf{R}$ & $\mathbf{R}$ & $\mathbf{R}$ & $\mathrm{S}$ & $\mathbf{R}$ & $\mathbf{R}$ & $\mathrm{S}$ & $\mathrm{S}$ & $\mathrm{R}$ \\
\hline Color of colonies ${ }^{b}$ & $P$ & L-Y & $\mathbf{Y}$ & $\mathbf{P}$ & W-Y & $P$ & Y-G & $\mathrm{P}$ & $\mathrm{P}$ & $\mathrm{P}$ & $P$ & $\mathrm{P}$ & $\mathrm{P}$ & $\mathbf{P}$ & L-Y \\
\hline Catalase activity & $+^{c}$ & + & + & + & + & + & + & + & + & + & + & + & + & + & + \\
\hline Oxidase activity & - & - & - & - & - & - & - & - & - & - & - & - & - & - & - \\
\hline \multicolumn{16}{|c|}{ Growth in the presence of: } \\
\hline $2.0 \% \mathrm{NaCl}$ & ++ & ++ & ++ & ++ & ++ & ++ & ++ & ++ & ++ & ++ & ++ & ++ & ++ & ++ & ++ \\
\hline $5.0 \% \mathrm{NaCl}$ & + & + & + & + & + & ++ & + & + & + & ++ & ++ & + & + & + & + \\
\hline $7.5 \% \mathrm{NaCl}$ & + & $+w$ & $+w$ & + & + & ++ & + & + & + & $+w$ & + & + & + & + & $+\mathrm{w}$ \\
\hline $10.0 \% \mathrm{NaCl}$ & $+w$ & - & - & $+w$ & $+\mathrm{w}$ & ++ & $+w$ & $+w$ & + & $+w$ & + & $+w$ & $+w$ & $+w$ & - \\
\hline $15.0 \% \mathrm{NaCl}$ & $+w$ & - & - & $+w$ & - & + & $+\mathrm{w}$ & $+\mathrm{w}$ & $+\mathrm{w}$ & $+w$ & $+w$ & $+\mathrm{w}$ & $+w$ & $+w$ & - \\
\hline Growth at $\mathrm{pH} 5.0$ & - & + & + & - & + & + & + & + & + & - & + & + & - & + & - \\
\hline \multicolumn{16}{|l|}{ Hydrolysis of: } \\
\hline Gelatin & - & - & - & - & + & $+w$ & - & $+w$ & - & - & - & - & - & - & - \\
\hline Starch & + & + & + & + & + & + & + & + & + & + & + & + & + & $\mathbf{w}$ & + \\
\hline Esculin & + & + & + & + & + & + & + & + & + & + & + & $+\mathrm{w}$ & + & + & + \\
\hline Tween 20 & - & - & - & - & - & - & - & - & - & - & - & - & - & - & - \\
\hline Tween 40 & - & - & - & - & - & - & - & - & - & - & - & - & - & - & - \\
\hline Tween 60 & - & - & - & - & - & - & - & - & - & $+w$ & + & $+w$ & $+w$ & - & $+w$ \\
\hline Tween 80 & - & - & - & - & - & - & - & - & - & + & + & $+w$ & $+w$ & - & $+\mathrm{w}$ \\
\hline Urease production & - & - & - & + & + & $+\mathrm{w}$ & + & + & + & + & + & + & + & + & + \\
\hline Nitrate reduction & + & + & + & + & + & + & + & + & + & + & + & + & + & + & + \\
\hline $\mathrm{H}_{2} \mathrm{~S}$ production & - & + & + & + & + & + & + & - & - & + & + & + & + & + & + \\
\hline Voges-Proskauer test & - & - & - & - & - & - & + & - & - & + & + & - & - & - & + \\
\hline \multirow{2}{*}{\multicolumn{16}{|c|}{ Acid production from: }} \\
\hline & & & & & & & & & & & & & & & \\
\hline D-Arabinose & $+w$ & - & - & - & - & - & - & - & - & - & - & $+w$ & - & - & - \\
\hline L-Arabinose & $+w$ & + & + & + & + & + & + & + & + & + & + & $+w$ & $+w$ & + & + \\
\hline Cellobiose & - & - & + & $+w$ & + & - & $+w$ & - & $+w$ & - & $+w$ & - & - & - & - \\
\hline D-Fructose & - & - & - & + & + & + & + & + & + & - & + & - & - & - & + \\
\hline D-Galactose & - & + & + & + & + & + & + & + & + & + & + & - & + & + & + \\
\hline D-Glucose & + & + & + & + & + & + & + & + & + & + & + & + & + & + & + \\
\hline Lactose & - & + & + & $+w$ & - & - & $+w$ & $+w$ & $+w$ & - & $+w$ & - & - & $\rightarrow$ & + \\
\hline Maltose & + & + & + & + & + & + & + & + & + & - & + & - & + & + & + \\
\hline D-Mannose & - & - & - & + & + & + & + & + & + & - & + & - & - & - & - \\
\hline Melezitose & $+w$ & - & - & $+w$ & + & + & - & - & - & - & $+w$ & - & - & - & - \\
\hline Raffinose & - & - & + & - & + & - & - & + & - & - & - & - & - & - & - \\
\hline L-Rhamnose & - & - & - & - & - & - & - & + & - & - & - & - & - & - & - \\
\hline D-Ribose & $+w$ & + & $+w$ & - & $+w$ & - & - & - & - & - & + & $+w$ & - & + & + \\
\hline Salicin & - & + & + & + & + & - & + & + & + & - & + & - & - & + & + \\
\hline Sucrose & - & - & + & + & + & + & + & + & + & - & + & - & - & - & - \\
\hline Trehalose & - & - & - & - & + & - & - & - & - & - & - & - & - & - & - \\
\hline D-Xylose & - & - & + & - & + & + & - & + & - & + & - & - & - & - & - \\
\hline Adonitol & - & - & - & + & + & - & + & - & - & - & - & - & - & - & + \\
\hline Inositol & - & - & - & + & + & - & - & - & - & - & - & - & - & + & - \\
\hline Mannitol & - & - & - & - & - & - & - & - & - & - & - & - & - & - & - \\
\hline Sorbitol & - & - & - & - & + & - & - & - & - & - & - & - & - & - & - \\
\hline Xylitol & - & - & - & - & - & - & - & - & - & - & - & - & - & - & - \\
\hline
\end{tabular}

${ }^{a} \mathrm{R}$, rapid; $\mathrm{S}$, slow.

${ }^{b} \mathrm{P}$, pale brown; L-Y, light yellow; W-Y, whitish yellow; Y-G, yellow-green; Y, yellow.

$c++$, strongly positive; + , positive; $+w$, weakly positive; - , negative.

2511, CCM 2589, CCM 2590, and CCM 2432), $M$. roseus NCTC 7281, and Brachybacterium sp. strain CCM 4385. " $M$. conglomeratus" CCM 2432 exhibited a high level of homology with the type strain of $B$. nesterenkovii, IMV Ac-752.

\section{DISCUSSION}

On the basis of biochemical and chemical criteria, the 15 strains which we studied were found to belong to the genus Brachybacterium.

Schleifer and Lang (16) reported that the cell morphology of " $M$. conglomeratus" strains is uniform and that only coccoid cells occur throughout the various phases of growth. In our study, we found that stationary-phase cultures of the 15 strains which we studied contained only coccoid cells and that earlylog-phase cultures contained rod-shaped cells.

Collins et al. (2) placed strains CCM 9860 ${ }^{\mathrm{T}}, \mathrm{CCM} 9859$, and CCM 9861 in the species B. faecium on the basis of their physiological and chemotaxonomic characteristics. On the other hand, Schleifer and Lang (16) reported that the levels of DNA-DNA homology between B. faecium CCM $9860^{\mathrm{T}}$ (= IFO $\left.14762^{\mathrm{T}}\right)$ and the other two $B$. faecium strains, CCM 9861 and CCM 9859, revealed moderate levels of relatedness (60 to 70 and 40 to $60 \%$, respectively), and they suggested that a level of 
TABLE 3. Chemotaxonomic characteristics

\begin{tabular}{|c|c|c|c|c|c|c|c|c|c|c|c|c|c|c|}
\hline \multirow{3}{*}{ Species } & \multirow{3}{*}{ Strain } & \multirow{3}{*}{$\begin{array}{l}\text { Mena- } \\
\text { quinone }\end{array}$} & \multirow{3}{*}{$\begin{array}{l}\text { Acyl } \\
\text { type }\end{array}$} & \multirow{3}{*}{$\begin{array}{l}\text { Mycolic } \\
\text { acid }\end{array}$} & \multirow{3}{*}{$\begin{array}{l}\text { Phospho- } \\
\text { lipid } \\
\text { profile }^{a}\end{array}$} & \multirow{3}{*}{$\begin{array}{l}\mathrm{G}+\mathrm{C} \\
\text { content } \\
\text { (mol\%) }\end{array}$} & \multicolumn{8}{|c|}{ Cell wall composition ${ }^{b}$} \\
\hline & & & & & & & \multicolumn{4}{|c|}{ Sugars } & \multicolumn{4}{|c|}{ Amino acids } \\
\hline & & & & & & & $\mathrm{Gal}$ & Glc & Rha & 6Tal & meso-DAP & Glu & Gly & Ala \\
\hline B. faecium & NCIB $9860^{\mathrm{T}}$ & MK-7 & Glycolyl & - & A & 72.1 & 1.0 & 0.32 & & 0.46 & 1.0 & 2.46 & 1.28 & 2.00 \\
\hline \multirow[t]{2}{*}{ B. nesterenkovii } & IMV Ac- $752^{\mathrm{T}}$ & MK-7 & Glycolyl & - & B & 72.0 & 1.0 & 0.45 & 0.53 & & 1.0 & 1.61 & & 1.61 \\
\hline & CCM 2432 & MK-7 & Glycolyl & - & B & 71.4 & 1.0 & 0.28 & 0.66 & & 1.0 & 2.35 & & 2.32 \\
\hline B. paraconglomeratum & NCIB $9861^{T}$ & MK-7 & Glycolyl & - & $\mathrm{C}$ & 68.6 & 1.0 & 0.49 & & & 1.0 & 3.22 & 1.31 & 2.22 \\
\hline B. rhamnosum & $\mathrm{H}-6 \mathrm{~S}^{\mathrm{T}}$ & MK-7 & Glycolyl & - & $\mathrm{D}$ & 71.5 & 1.0 & 0.76 & 4.52 & & 1.0 & 2.57 & 0.88 & 1.96 \\
\hline \multirow[t]{10}{*}{ B. conglomeratum } & NCIB 9859 & MK-7 & Glycolyl & - & $\mathrm{E}$ & 70.5 & 1.0 & 0.69 & & & 1.0 & 3.34 & 1.21 & 2.12 \\
\hline & CCM 4385 & MK-7 & Glycolyl & - & $\mathrm{E}$ & 71.2 & 1.0 & 0.47 & & & 1.0 & 2.56 & 1.53 & 3.12 \\
\hline & CCM 2135 & MK-7 & Glycolyl & - & $\mathrm{F}$ & 70.8 & 1.0 & 0.50 & & & 1.0 & 2.07 & 1.35 & 2.70 \\
\hline & CCM 2134 & MK-7 & Glycolyl & - & $\mathrm{F}$ & 71.4 & 1.0 & 0.53 & & & 1.0 & 1.22 & 0.78 & 1.49 \\
\hline & CCM 2136 & MK-7 & Glycolyl & - & $\mathrm{E}$ & 71.6 & 1.0 & 0.42 & & & 1.0 & 1.82 & 1.00 & 1.75 \\
\hline & CCM 2137 & MK-7 & Glycolyl & - & E & 72.6 & 1.0 & 0.22 & & & 1.0 & 2.32 & 1.23 & 2.32 \\
\hline & CCM 2511 & MK-7 & Glycolyl & - & $\mathrm{E}$ & 70.2 & 1.0 & 0.44 & & & 1.0 & 1.83 & 1.47 & 2.78 \\
\hline & CCM $2589^{\mathrm{T}}$ & MK-7 & Glycolyl & - & $\mathrm{E}$ & 70.6 & 1.0 & 0.42 & & & 1.0 & 2.69 & 1.63 & 2.86 \\
\hline & CCM 2590 & MK-7 & Glycolyl & - & $\bar{E}$ & 70.9 & 1.0 & 0.21 & & & 1.0 & 2.08 & 1.12 & 2.00 \\
\hline & NCTC 7281 & MK-7 & Glycolyl & - & E & 71.5 & 1.0 & 0.20 & & & 1.0 & 2.51 & 1.40 & 2.86 \\
\hline
\end{tabular}

${ }^{a}$ See Fig. 2.

${ }^{b} \mathrm{Gal}$, galactose; Glc, glucose; Rha, rhamnose; 6Tal, 6-deoxytalose; Glu, glutamic acid; Gly, glycine; Ala, alanine.

DNA-DNA homology of about $65 \%$ represents the borderline value for subspecies differentiation. In our study, the levels of DNA-DNA homology among these strains were less than $50 \%$, and therefore, we concluded that they should be classified as members of separate species. Our dendrogram based on the quantitative fatty acid data (Fig. 3) also showed that $B$. faecium CCM $9860^{\mathrm{T}}$, CCM 9861, and CCM 9859 should be separated from each other.

On the basis of morphological, physiological, and chemotaxonomic characteristics and DNA-DNA hybridization data, we describe three new Brachybacterium species: Brachybacterium conglomeratum sp. nov., nom. rev. (type strain, CCM 9860 [= IFO 15472]) for strains CCM 9860, NCIB 9859, CCM 2134, CCM 2135, CCM 2136, CCM 2137, CCM 2511, CCM 2589, CCM 2590, CCM 4385, and NCTC 7281; Brachybacterium paraconglomeratum sp. nov. (type strain, NCIB 9861 [= IFO 15224]) for strain NCIB 9861 (formerly a B. faecium strain); and Brachybacterium rhamnosum sp. nov. (type strain, H-6S [= IFO 15203]) for strain $\mathrm{H}-6 \mathrm{~S}$, which was isolated from corn steep liquor. Strain NCTC 7281 , which was received as $M$. roseus, is not a type strain and is not an $M$. roseus strain; this strain was identified as a member of $B$. conglomeratum in this study.

Differential characteristics of these new Brachybacterium species are shown in Table 6.

The physiological characteristics of $B$. paraconglomeratum and $B$. conglomeratum are similar, but these taxa can be distinguished on the basis of their cellular fatty acid types (Fig. 3 ) and low DNA-DNA hybridization values. B. rhamnosum is characterized by the presence of large amounts of rhamnose in its cell wall, and $B$. nesterenkovii is characterized by the absence of glycine in its cell wall. B. nesterenkovii IMV Ac-752 ${ }^{\mathrm{T}}$ and CCM 2432 were included in the same cluster as $B$. conglomeratus and $B$. rhamnosum on the dendrogram based on quantitative fatty acid data (Fig. 3), but these two strains are quite different from the other strains in the cluster in their chemotaxonomic characteristics (e.g., cell wall sugars and phospholipid type) and DNA-DNA homology values. $B$. faecium NCIB $9860^{\mathrm{T}}$ is characterized by the presence of 6 -deoxytalose in its cell wall.

TABLE 4. Cellular fatty acid compositions

\begin{tabular}{|c|c|c|c|c|c|c|c|c|c|c|c|c|c|}
\hline \multirow[b]{2}{*}{ Species } & \multirow[b]{2}{*}{ Strain } & \multicolumn{12}{|c|}{$\%$ of total fatty acids } \\
\hline & & $\begin{array}{l}\text { iso- } \\
14: 0\end{array}$ & 14:0 & $\begin{array}{l}\text { iso- } \\
15: 0\end{array}$ & $\begin{array}{c}\text { anteiso- } \\
15: 0\end{array}$ & 15:0 & $15: 1$ & $\begin{array}{l}\text { iso- } \\
16: 0\end{array}$ & 16:0 & $\begin{array}{l}\text { iso- } \\
17: 0\end{array}$ & $\begin{array}{c}\text { anteiso- } \\
17: 0\end{array}$ & $18: 0$ & $18: 1$ \\
\hline B. faecium & NCIB $9860^{\mathrm{T}}$ & 0.8 & & 18.8 & 58.1 & & 2.7 & 3.2 & & 2.2 & 10.1 & 4.0 & \\
\hline \multirow[t]{2}{*}{ B. nesterenkovii } & IMV Ac- $752^{\mathrm{T}}$ & 3.1 & 2.9 & 3.7 & 42.3 & & 2.9 & 11.1 & & & 12.4 & 16.9 & 4.5 \\
\hline & CCM 2432 & 1.6 & 2.7 & 4.0 & 49.6 & & 3.1 & 12.3 & & & 17.2 & 7.1 & 2.4 \\
\hline B. paraconglomeratum & NCIB $9861^{T}$ & 0.9 & 2.1 & 2.8 & 57.1 & 5.8 & 4.1 & 6.9 & 6.4 & 0.9 & 5.1 & 4.6 & 3.1 \\
\hline B. rhamnosum & $\mathrm{H}-6 \mathrm{~S}^{\mathrm{T}}$ & 1.9 & 1.0 & & 48.2 & 13.0 & 9.2 & 3.0 & 17.0 & & 6.1 & 0.5 & \\
\hline \multirow{10}{*}{ B. conglomeratum } & NCIB 9859 & 2.5 & 1.9 & 4.6 & 61.6 & 4.6 & 12.2 & 1.9 & & 1.8 & 9.0 & & \\
\hline & CCM 4385 & 1.1 & 0.9 & 10.6 & 41.7 & 6.3 & 14.2 & 2.6 & 2.9 & 3.5 & 15.5 & 0.7 & \\
\hline & CCM 2135 & 0.8 & 0.7 & 12.7 & 39.9 & & 11.4 & 5.0 & & & 26.9 & 2.5 & \\
\hline & CCM 2134 & 1.0 & 0.9 & 4.1 & 28.5 & & 18.6 & 10.5 & & 2.9 & 25.3 & 8.3 & \\
\hline & CCM 2136 & 0.7 & 0.6 & 5.0 & 30.1 & & 12.4 & 7.1 & & 6.2 & 36.7 & 1.3 & \\
\hline & CCM 2137 & 1.1 & 0.9 & 2.9 & 29.2 & & 21.8 & 9.1 & & 2.6 & 28.6 & 3.7 & \\
\hline & CCM 2511 & 1.7 & 0.9 & 4.3 & 57.9 & & 8.1 & 5.4 & & 1.4 & 14.2 & 6.2 & \\
\hline & CCM $2589^{\mathrm{T}}$ & 2.2 & 1.4 & 10.6 & 48.9 & & 11.2 & 7.3 & & 1.2 & 8.5 & 8.5 & \\
\hline & CCM 2590 & 1.7 & 0.9 & 2.7 & 28.8 & & 22.6 & 9.0 & & 2.2 & 27.5 & 4.6 & \\
\hline & NCTC 7281 & 1.4 & 0.7 & 7.9 & 47.1 & & 13.4 & 5.5 & & 1.4 & 18.5 & 4.0 & \\
\hline
\end{tabular}



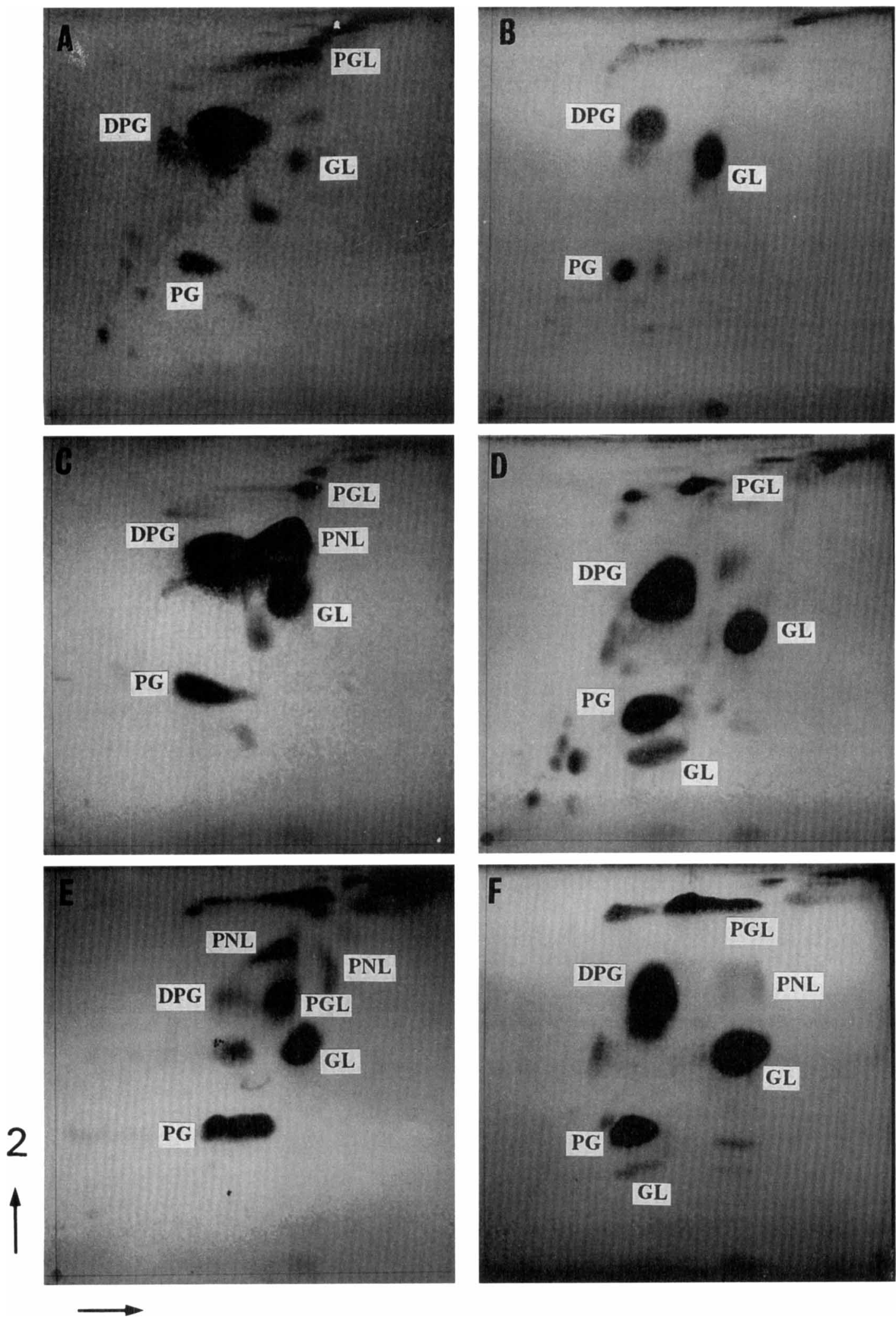

FIG. 2. Two-dimensional thin-layer chromatograms of polar lipids from B. faecium $\mathrm{NCIB} 9860^{\mathrm{T}}$ (A), B. nesterenkovii IMV Ac-752 ${ }^{\mathrm{T}}$ (B), B. paraconglomeratum NCIB $9861^{\mathrm{T}}$ (C), B. rhamnosum $\mathrm{H}-6 \mathrm{~S}^{\mathrm{T}}$ (D), B. conglomeratum $\mathrm{CCM} 2589^{\mathrm{T}}$ (E), and B. conglomeratum CCM $2134(\mathrm{~F})$. Chloroform-methanol-water $(65: 25: 4)$ was used in the first dimension, and chloroform-methanol-acetate-water (80:12:15:4) was used in the second dimension. Lipids were revealed by spraying the chromatograms with $10 \%$ molybdophosphoric acid in ethanol. Abbreviations: DPG, diphosphatidylglycerol; PG, phosphatidylglycerol; GL, glycolipid; PGL, ninhydrin-negative unknown phosphoglycolipid; PNL, ninhydrin-positive unknown phosphoglycolipid. 


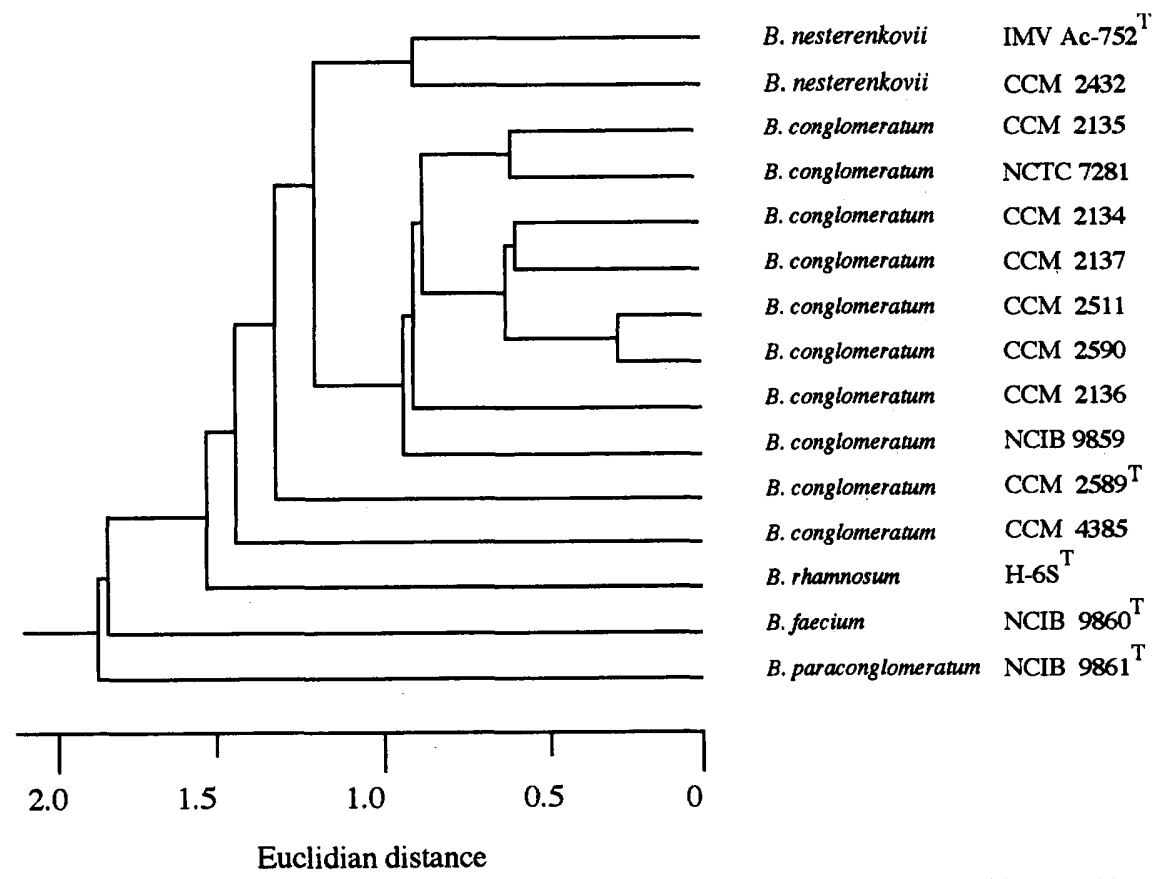

FIG. 3. Dendrogram showing the relationships among the 15 strains studied. The dendrogram was based on fatty acid composition data. Data were analyzed by using the NTSYS-pc program package (12), and clustering was performed by using the unweighted pair group arithmetic average method.

The new Brachybacterium species are described below.

Description of Brachybacterium conglomeratum sp. nov., nom. rev. Brachybacterium conglomeratum (con.glo.me.ra' tum. L. part. adj. conglomeratum, rolled together) is gram positive, nonmotile, and nonsporing. In the stationary growth phase, cells are coccoid; however, when a culture is transferred to fresh medium, the cells become oval or rod shaped. Colonies are circular, entire, low convex, smooth, opaque, and whitish yellow or pale brown. Aerobic or facultatively anaerobic. Catalase positive and oxidase negative. Grows well in the presence of $7.5 \% \mathrm{NaCl}$ and weakly in the presence of 10 to $15 \% \mathrm{NaCl}$. Some strains grow at $\mathrm{pH}$ 5.0. Starch, esculin, and urea are hydrolyzed, but Tween 20 and Tween 40 are not hydrolyzed. Some strains hydrolyze Tween 60 and Tween 80 . Nitrate is reduced to nitrite. Acid is produced from L-arabinose, D-galactose, and D-glucose. Acid is not produced from trehalose, mannitol, sorbitol, and xylitol.

The cell wall peptidoglycan is based on meso-DAP. The cell wall sugars are galactose and glucose. The cell wall muramic acid occurs in the $N$-glycolyl form. Mycolic acids are absent. The principal menaquinone is MK-7. The long-chain fatty acids are primarily straight-chain, saturated, iso and anteiso methyl-branched fatty acids; anteiso- $C_{15: 0}$ and anteiso- $C_{17: 0}$ are the predominant fatty acids. The major polar phospholip-

TABLE 5. DNA-DNA homology values

\begin{tabular}{|c|c|c|c|c|c|c|c|c|c|c|c|}
\hline \multirow{3}{*}{ Species } & \multirow{3}{*}{ Strain } & \multicolumn{10}{|c|}{ \% DNA-DNA reassociation with: } \\
\hline & & \multirow{2}{*}{$\begin{array}{l}\text { B. faecium } \\
\text { NCIB } \\
9860^{\mathrm{T}}\end{array}$} & \multicolumn{2}{|c|}{$\begin{array}{c}\text { B. nesterenkovii } \\
\text { strains }\end{array}$} & \multirow{2}{*}{$\begin{array}{c}\text { B. paracon- } \\
\text { glomeratum } \\
\text { NCIB } \\
9861^{\mathrm{T}}\end{array}$} & \multirow{2}{*}{$\begin{array}{c}\text { B. rhamnosum } \\
\text { H-6S }\end{array}$} & \multicolumn{5}{|c|}{ B. conglomeratum strains } \\
\hline & & & $\begin{array}{c}\text { IMV } \\
\text { Ac-752 }^{\mathrm{T}}\end{array}$ & $\begin{array}{r}\text { CCM } \\
2432\end{array}$ & & & $\begin{array}{l}\text { NCIB } \\
9859\end{array}$ & $\begin{array}{l}\mathrm{CCM} \\
4385\end{array}$ & $\begin{array}{l}\text { CCM } \\
2135\end{array}$ & $\begin{array}{l}\text { CCM } \\
2136\end{array}$ & $\begin{array}{l}\mathrm{CCM} \\
2589^{\mathrm{T}}\end{array}$ \\
\hline B. faecium & NCIB $9860^{\mathrm{T}}$ & 100 & 5 & 18 & 19 & 28 & 17 & 26 & 27 & 28 & 19 \\
\hline \multirow[t]{2}{*}{ B. nesterenkovii } & IMV Ac- $752^{\mathrm{T}}$ & 5 & 100 & 98 & 21 & 7 & 5 & 5 & 3 & 20 & 13 \\
\hline & CCM 2432 & 27 & 92 & 100 & 32 & 6 & 24 & 31 & 35 & 38 & 17 \\
\hline B. paraconglomeratum & NCIB $9861^{\mathrm{T}}$ & 19 & 12 & 19 & 100 & 11 & 13 & 14 & 14 & 14 & 10 \\
\hline B. rhamnosum & $H-6 S^{\mathrm{T}}$ & 11 & 3 & 13 & 5 & 100 & 9 & 9 & 8 & 31 & 22 \\
\hline \multirow[t]{10}{*}{ B. conglomeratum } & NCIB 9859 & 49 & 19 & 23 & 31 & 32 & 100 & 125 & 103 & 104 & 76 \\
\hline & CCM 4385 & 12 & 7 & 31 & 17 & 11 & 78 & 100 & 73 & 66 & $\mathrm{NT}^{a}$ \\
\hline & CCM 2135 & 14 & 10 & 36 & 10 & 9 & 71 & 61 & 100 & 71 & NT \\
\hline & CCM 2134 & 9 & 8 & 2 & 16 & 7 & 61 & 57 & 76 & 69 & NT \\
\hline & CCM 2136 & 30 & 26 & 5 & 20 & 34 & 76 & 59 & 83 & 100 & 95 \\
\hline & CCM 2137 & 32 & 20 & 8 & 26 & 23 & 87 & 95 & 105 & 98 & 79 \\
\hline & CCM 2511 & 12 & 15 & 30 & 17 & 38 & 66 & 95 & 83 & 70 & 69 \\
\hline & CCM $2589^{\mathrm{T}}$ & 12 & 10 & 14 & 19 & 17 & 70 & 87 & 76 & 82 & 100 \\
\hline & CCM 2590 & 22 & 14 & 18 & 17 & 12 & 93 & 81 & 99 & 79 & NT \\
\hline & NCTC 7281 & 25 & 16 & 34 & 26 & 17 & 83 & 96 & 94 & 80 & 64 \\
\hline
\end{tabular}

${ }^{a} \mathrm{NT}$, not tested. 
TABLE 6. Differential characteristics of five Brachybacterium species

\begin{tabular}{|c|c|c|c|c|c|}
\hline Characteristic & $\begin{array}{l}\text { B. faecium } \\
(n=1)^{a}\end{array}$ & $\begin{array}{l}\text { B. nesterenkovii } \\
\quad(n=2)\end{array}$ & $\begin{array}{l}\text { B. paraconglomeratum } \\
\qquad(n=1)\end{array}$ & $\begin{array}{l}\text { B. rhamnosum } \\
(n=1)\end{array}$ & $\begin{array}{l}\text { B. conglomeratum } \\
(n=10)\end{array}$ \\
\hline \multicolumn{6}{|c|}{$\begin{array}{l}\text { Morphological, physiological, and biochemical } \\
\text { characteristics }\end{array}$} \\
\hline Growth rate ${ }^{b}$ & $\mathrm{~S}$ & $\mathrm{R}$ & $\mathrm{S}$ & $\mathrm{R}$ & $\mathrm{R}, \mathrm{S}$ \\
\hline Color of colonies ${ }^{c}$ & $\mathbf{P}$ & $\mathrm{L}-\mathrm{Y}, \mathrm{Y}$ & $\mathbf{P}$ & W-Y & $\mathrm{P}, \mathrm{Y}-\mathrm{G}, \mathrm{L}-\mathrm{Y}$ \\
\hline \multicolumn{6}{|l|}{ Growth in the presence of: } \\
\hline $7.5 \% \mathrm{NaCl}$ & $+^{d}$ & $+\mathrm{w}$ & + & + & 100 \\
\hline $10.0 \% \mathrm{NaCl}$ & $+\mathrm{w}$ & - & $+w$ & $+w$ & 90 \\
\hline $15.0 \% \mathrm{NaCl}$ & $+w$ & - & $+w$ & - & 90 \\
\hline Growth at $\mathrm{pH} 5.0$ & - & + & - & + & 70 \\
\hline Hydrolysis of gelatin & - & - & - & + & 20 \\
\hline Hydrolysis of Tween 60 & - & - & - & - & 50 \\
\hline Hydrolysis of Tween 80 & - & - & - & - & 50 \\
\hline Urease production & - & - & + & + & 100 \\
\hline $\mathrm{H}_{2} \mathrm{~S}$ production & - & + & + & + & 80 \\
\hline Voges-Proskauer test & - & - & - & - & 40 \\
\hline Indole formation & $+\mathrm{w}$ & - & + & - & 90 \\
\hline \multicolumn{6}{|l|}{ Acid production from: } \\
\hline D-Arabinose & $+\mathrm{w}$ & - & - & - & 10 \\
\hline Cellobiose & - & $\mathrm{d}$ & $+\mathrm{w}$ & + & 30 \\
\hline D-Fructose & - & - & + & + & 60 \\
\hline D-Galactose & - & + & + & + & 90 \\
\hline Lactose & - & + & $+\mathrm{w}$ & - & 50 \\
\hline D-Mannose & - & - & + & + & 50 \\
\hline Melezitose & $+\mathrm{w}$ & - & $+\mathrm{w}$ & + & 20 \\
\hline Raffinose & - & $\mathrm{d}$ & - & + & 10 \\
\hline D-Ribose & $+\mathrm{w}$ & + & - & $+\mathrm{w}$ & 40 \\
\hline Salicin & - & + & + & + & 60 \\
\hline Sucrose & - & - & + & + & 50 \\
\hline Trehalose & - & - & - & + & 0 \\
\hline D-Xylose & - & $\mathrm{d}$ & - & + & 30 \\
\hline Adonitol & - & - & + & + & 20 \\
\hline Inositol & - & - & + & + & 10 \\
\hline Sorbitol & - & - & - & + & 0 \\
\hline \multicolumn{6}{|l|}{ Chemotaxonomic characteristics } \\
\hline Cell wall contains rhamnose & - & + & - & ++ & 0 \\
\hline Cell wall contains 6-deoxy-L-talose & + & - & - & - & 0 \\
\hline Cell wall contains glycine & + & - & + & + & 100 \\
\hline Phospholipid type(s) ${ }^{e}$ & A & B & $\mathrm{C}$ & $\mathrm{D}$ & $\mathrm{E}, \mathrm{F}$ \\
\hline
\end{tabular}

${ }^{a} n$ is the number of strains examined.

${ }^{b} \mathrm{R}$, rapid; $\mathrm{S}$, slow.

${ }^{c}$ P, pale brown; L-Y, light yellow; Y, yellow; W-Y, whitish yellow; Y-G, yellow-green.

$d+$, positive; ++ , strongly positive; $+\mathrm{w}$, weakly positive; - , negative; $\mathrm{d}$, different reactions in different strains. The values are the percentages of strains that are positive.

${ }^{e}$ See Fig. 2.

ids are diphosphatidylglycerol, phosphatidylglycerol, unidentified glycolipids, and unidentified, ninhydrin-positive phosphoglycolipids. The $\mathrm{G}+\mathrm{C}$ content of the DNA is 70.2 to 71.6 mol\%; the $\mathrm{G}+\mathrm{C}$ content of the type strain is $70.6 \mathrm{~mol} \%$.

The type strain is CCM 2589 (= IFO 15472).

Description of Brachybacterium paraconglomeratum sp. nov. Brachybacterium paraconglomeratum (pa.ra.con.glo.me.ra'tum. L. part. adj. para, alongside of, resembling; M.L. adj. conglomeratum, specific epithet of B. conglomeratum; M.L. gen. $\mathrm{n}$. paraconglomeratum, resembling $B$. conglomeratum) is gram positive, nonmotile, and nonsporing. In the stationary growth phase, cells are coccoid; however, when a culture is transferred to fresh medium, the cells become oval or rod shaped. Colonies are circular, entire, low convex, smooth, and opaque, and the colony color is pale brown. Aerobic or facultatively anaerobic. Catalase positive and oxidase negative. Grows well in the presence of $7.5 \% \mathrm{NaCl}$. Does not grow at $\mathrm{pH}$ 5.0. Starch, esculin, and urea are hydrolyzed, but Tween 20, Tween 40, Tween 60, and Tween 80 are not hydrolyzed. $\mathrm{H}_{2} \mathrm{~S}$ and indole are produced. Nitrate is reduced to nitrite. Acid is produced from many sugars.

The cell wall peptidoglycan is based on meso-DAP. The cell wall sugars are galactose and glucose.

The cell wall muramic acid occurs in the $N$-glycolyl form. Mycolic acids are absent. The principal menaquinone is MK-7. The long-chain fatty acids are primarily straight-chain, saturated, iso and anteiso methyl-branched fatty acids; anteiso$\mathrm{C}_{15: 0}$ and anteiso- $\mathrm{C}_{17: 0}$ are the predominant fatty acids. The major polar phospholipids are diphosphatidylglycerol, phosphatidylglycerol, unidentified glycolipids, and unidentified, ninhydrin-positive phosphoglycolipids. The $\mathrm{G}+\mathrm{C}$ content of the DNA is $68.6 \mathrm{~mol} \%$.

The type strain is NCIB 9861 (= IFO 15224).

Description of Brachybacterium rhamnosum sp. nov. Brachybacterium rhamnosum (rham.nos'um. L. n. rhamnosum, rhamnose, referring to the abundance of rhamnose in the cell wall) is gram positive, nonmotile, and nonsporing. In the stationary growth phase, cells are coccoid; however, when a culture is 
transferred to fresh medium, the cells become oval or rod shaped. Colonies are circular, entire, low convex, smooth, opaque, and whitish yellow. Aerobic or facultatively anaerobic. Catalase positive and oxidase negative. Grows well in the presence of $7.5 \% \mathrm{NaCl}$. Grows at $\mathrm{pH}$ 5.0. Gelatin, starch, esculin, and urea are hydrolyzed, but Tween 20 , Tween 40 , Tween 60, and Tween 80 are not hydrolyzed. Nitrate is reduced to nitrite. $\mathrm{H}_{2} \mathrm{~S}$ is produced. Acid is produced from many sugars.

The cell wall peptidoglycan is based on meso-DAP. The cell wall sugars are galactose, glucose, and rhamnose. The cell wall muramic acid occurs in the $N$-glycolyl form. Mycolic acids are absent. The principal menaquinone is MK-7. The long-chain fatty acids are primarily straight-chain, saturated, iso and anteiso methyl-branched fatty acids; anteiso- $\mathrm{C}_{15: 0}$ and anteiso$\mathrm{C}_{17: 0}$ are the predominant fatty acids. The major polar phospholipids are diphosphatidylglycerol, phosphatidylglycerol, and unidentified glycolipids. The $\mathrm{G}+\mathrm{C}$ content of the DNA is $71.5 \mathrm{~mol} \%$. Isolated from commercially available corn steep liquor.

The type strain is $\mathrm{H}-6 \mathrm{~S}$ (= IFO 15203).

\section{ACKNOWLEDGMENTS}

We thank Toru Hasegawa, Institute for Fermentation, Osaka, for support and discussions.

C.-X.F. is indebted to the Matsumae International Foundation, Tokyo, Japan, for research and maintenance grants.

\section{REFERENCES}

1. Bogdanovsky, D., E. Interschick-Niebler, K.-H. Schleifer, F. Fiedler, and $\mathbf{O}$. Kandler. 1971. $\gamma$-Glutamyl-glutamic acid, an interpeptide bridge in the murein of some micrococci and Arthrobacter sp. Eur. J. Biochem. 22:173178 .

2. Collins, M., J. Brown, and D. Jones. 1988, Brachybacterium faecium gen. nov., sp. nov., a coryneform bacterium from poultry deep litter. Int. J. Syst. Bacteriol. 38:45-48.

3. Cowan, S. T., and K. J. Steel. 1965. Manual for the identification of medical bacteria. Cambridge University Press, London.

4. Ezaki, T., Y. Hashimoto, and E. Yabuuchi. 1989. Fluorometric deoxyribonucleic acid-deoxyribonucleic acid hybridization in microdilution wells as an alternative to membrane filter hybridization in which radioisotopes are used to determine genetic relatedness among bacterial strains. Int. J. Syst. Bacteriol. 39:224-229.

5. Gvozdyak, O. R., T. M. Nogina, and P. Schumann. 1992. Taxonomic study of the genus Brachybacterium: Brachybacterium nesterenkovii sp. nov. Int. J. Syst. Bacteriol. 42:74-78.

6. Iizuka, H., and K. Komagata. 1965. Microbiological studies on petroleum and natural gas. III. Determination of Brevibacterium, Arthrobacter, Micrococcus, Sarcina, Alcaligenes, and Achromobacter isolated from oil-brines in Japan. J. Gen. Appl. Microbiol. 11:1-14.

7. Mesbah, M., U. Premachandran, and W. B. Whitman. 1989. Precise measurement of the $\mathrm{G}+\mathrm{C}$ content of deoxyribonucleic acid by highperformance liquid chromatography. Int. J. Syst. Bacteriol, 39:159-167.

8. Mikami, H., and Y. Ishida. 1993. Post-column fluorometric detection of reducing sugars in high-performance liquid chromatography using arginine. Bunseki Kagaku 32:E207-E210.

9. Minnikin, D. E., L. Alshamaony, and M. Goodfellow. 1975. Differentiation of Mycobacterium, Nocardia and related taxa by thin-layer chromatographic analysis of whole-organism methanolysates. J. Gen. Microbiol. 88:200-206.

10. Minnikin, D. E., M. D. Collins, and M. Goodfellow. 1979. Fatty acid and polar lipid composition in the classification of Cellulomonas, Oerskovia and related taxa. J. Appl. Bacteriol. 47:87-95.

11. Nogina, T. M., O. R. Gvozdyak, Y. S. Sadovnikov, V. V. Shkaruba, and S. L. Kuberskaya. 1991. Phenotypical study and numerical analysis of the strains of coryneform bacteria with A4y peptidoglycan variation. Mikrobiol. $\mathrm{Zh}$. (Kiev) 53:2-10. (In Russian.)

12. Rohlf, F. J. 1989. NTSYS-pc. Numerical taxonomy and multivariate analysis system, version 1.50. Exeter Publishing, Ltd., New York.

13. Saito, H., and K. Miura. 1963. Preparation of transforming deoxyribonucleic acid by phenol treatment. Biochim. Biophys. Acta 72:619-629.

14. Schefferle, H. E. 1966. Coryneform bacteria in poultry deep litter. J. Appl. Bacteriol. 29:147-160.

15. Schleifer, K. H., and O. Kandler. 1972. Peptidoglycan types of bacterial cell walls and their taxonomic implications. Bacteriol. Rev. 36:407-477.

16. Schleifer, K.-H., and K. Lang. 1980. Close relationship among strains of Micrococcus conglomeratus and Arthrobacter species. FEMS Microbiol. Lett. 9:223-226.

17. Suzuki, K., and K. Komagata. 1983. Taxonomic significance of cellular fatty acid composition in some coryneform bacteria. Int. J. Syst. Bacteriol. 33:188-200.

18. Uchida, K., and K. Aida. 1977. Acyl type of bacterial cell wall: its simple identification by a colorimetric method. J. Gen. Appl. Microbiol. 23:249-260.

19. Wako Pure Chemical Industries, Ltd. 1989. Technical note on the system of PTC-amino acid analysis. Wako Pure Chemical Industries, Ltd., Osaka, Japan. (In Japanese.)

20. Yokota, A., M. Takeauchi, and N. Weiss. 1993. Proposal of two new species in the genus Microbacterium: Microbacterium dextranolyticum sp. nov. and Microbacterium aurum sp. nov. Int. J. Syst. Bacteriol. 43:549-554. 